\title{
Absolute spectrophotometry of northern compact planetary nebulae ${ }^{\star}$
}

\author{
S. A. Wright ${ }^{1,2}$, R .L. M. Corradi ${ }^{1}$, and M. Perinotto ${ }^{3}$ \\ ${ }^{1}$ Isaac Newton Group of Telescopes, Ap. de Correos 321, 38700 Sta. Cruz de la Palma, Canary Islands, Spain \\ e-mail: rcorradi@ing.iac.es \\ 2 UCLA, Department of Physics and Astronomy, Los Angeles, CA, 90095, USA \\ e-mail: saw@astro.ucla.edu \\ 3 Dipartimento di Astronomia e Scienza dello Spazio, Universitá di Firenze, L.go E. Fermi 5, 50125 Firenze, Italy \\ e-mail: mariop@arcetri.astro.it
}

Received 19 November 2002 / Accepted 14 March 2005

\begin{abstract}
We present medium-dispersion spectra and narrowband images of six northern compact planetary nebulae (PNe): BoBn 1, DdDm 1, IC 5117, M 1-5, M 1-71, and NGC 6833. From broad-slit spectra, total absolute fluxes and equivalent widths were measured for all observable emission lines. High signal-to-noise emission line fluxes of $\mathrm{H} \alpha, \mathrm{H} \beta$, [OIII], [NII], and HeI may serve as emission line flux standards for northern hemisphere observers. From narrow-slit spectra, we derive systemic radial velocities. For four PNe, available emission line fluxes were measured with sufficient signal-to-noise to probe the physical properties of their electron densities, temperatures, and chemical abundances. BoBn 1 and DdDm 1, both type IV $\mathrm{PNe}$, have an $\mathrm{H} \beta$ flux over three sigma away from previous measurements. We report the first abundance measurements of M 171. NGC 6833 measured radial velocity and galactic coordinates suggest that it is associated with the outer arm or possibly the galactic halo, and its low abundance $\left([\mathrm{O} / \mathrm{H}]=1.3 \times 10^{-4}\right)$ may be indicative of low metallicity within that region.
\end{abstract}

Key words. ISM: planetary nebulae: general - techniques: spectroscopic - line: identification - ISM: abundances

\section{Introduction}

Measuring absolute emission-line fluxes of planetary nebulae $(\mathrm{PNe})$ is performed for a number of reasons. Primarily, absolute fluxes can be related to basic properties of the nebulae and their central stars (i.e. masses and luminosities, once distances are independently known), and can be used to determine nebular physico-chemical properties. If total flux of a $\mathrm{PNe}$ is accurately measured, monitoring over decades can also reveal possible luminosity variations, which in turn are related to fast evolution of the central star during the PNe phase, especially for the largest core masses.

In addition, absolute emission-line fluxes of PNe may also be used as standard calibrations for narrow band photometry. In fact, while highly precise broad band photometric (Johnson \& Harris 1954; Landolt 1992) and spectrophotometric (e.g., Stone \& Baldwin 1983; Massey et al. 1988; Oke 1990) standard stars are presently available, there is still a deficiency of emission-line flux standards, which is a requisite for high precision photometric study of ionized nebulae (PNe, HII regions,

\footnotetext{
* Based on observations obtained at the $2.5 \mathrm{~m}$ INT telescope of the Isaac Newton Group and the $2.6 \mathrm{~m}$ NOT telescope operated by NOTSA in the Observatorio del Roque de Los Muchachos, and with the NASA/ESA Hubble Space Telescope, obtained at the Space Telescope Science Institute, which is operated by AURA for NASA under contract NAS5-26555.
}

cataclysmic variables, supernova remnants, etc.). For narrow band photometry, broad band standards observed through a specific filter may not be easily related to the flux of an emission-line source. The reason is that the transmission curve of the filter is often not accurately known, as it depends on many parameters such as temperature during observing, tilt, focal ratio, axial angle within the instrument, and filter age. In addition, several emission lines coincide with absorption lines present in continuum standards (e.g. Balmer series), whose exact contribution in narrow band filters is difficult to estimate.

In the past, a number of emission-line standards were derived with use of photon counting detectors coupled with interference filters (Liller 1955; O'Dell 1963), but they have relatively large errors. In the northern hemisphere, standards primarily for $\mathrm{H} \beta$ fluxes were derived by calibrating compact $\mathrm{PNe}$ with spectrophotometric stars, by means of photoelectric scanning observations (e.g. Liller \& Aller 1954; Capriotti \& Daub 1960; Miller \& Mathews 1972; Barker 1978). Recently, to improve on similar conventional techniques in the southern hemisphere, Dopita \& Hua (1997) derived a set of southern emission-line standards by means of broad-slit absolute spectrophotometry of a sample of compact PNe. Similar emissionline standards are completely missing in the northern hemisphere.

Along these lines, we have selected a small sample of (supposedly) compact PNe observable from La Palma, and 
Table 1. Observational details of target PNe. Diameters are computed.

\begin{tabular}{lccccc}
\hline \hline $\begin{array}{l}\text { Object } \\
\text { Name }\end{array}$ & $\begin{array}{c}\text { PN G } \\
\text { Number }\end{array}$ & RA (J2000) Dec & $\begin{array}{c}\text { Diameter } \\
{\left[{ }^{\prime \prime}\right]}\end{array}$ & $\begin{array}{c}V_{\odot} \\
{\left[\mathrm{km} \mathrm{s}^{-1}\right]}\end{array}$ \\
\hline BoBn 1 & $108.4-76.1$ & 003718.0 & -134212 & $1.5 \times 2.2$ & $+174 \pm 10$ \\
DdDm 1 & $061.9+41.3$ & 164019.4 & +384203 & 1.4 & $-317 \pm 13$ \\
IC 5117 & $089.8-05.1$ & 213231.0 & +443548 & $1.6 \times 3.5$ & $-47 \pm 6$ \\
M 1-5 & $184.0-02.1$ & 054650.2 & +242202 & $2.3 \times 2.8$ & $+28 \pm 7$ \\
M 1-71 & $055.5-00.5$ & 193626.5 & +194220 & $3.7 \times 6.0$ & $+42 \pm 8$ \\
NGC 6833 & $082.5+11.3$ & 194946.6 & +485740 & $0.5 \times 0.6$ & $-110 \pm 2$ \\
\hline
\end{tabular}

attempted to measure their integrated emission-line fluxes. To make full use of the wide spectral coverage, we have measured absolute fluxes and equivalent widths for all emission lines present in our observations to investigate further the physical and chemical properties of these PNe. In Sect. 2 we explain the spectroscopic and narrowband imaging observational details. Section 3 discusses the morphology and sizes of these PNe, and Sect. 4 presents the analysis and results for fluxes, equivalent widths, radial velocities and chemical abundances. Discussion and comparison of our results to literature are made in Sect. 5.

\section{Observations and reduction}

\subsection{Spectroscopy}

Spectroscopic observations were obtained at the $2.5 \mathrm{~m}$ Isaac Newton Telescope (INT) of the Isaac Newton Group of Telescopes, Roque de los Muchachos, La Palma, using the Intermediate Dispersion Spectrograph (IDS). Each observation night was conducted under photometric conditions with seeing mostly between 1 and 1.5 arcsec, and only rarely as high as 2.5 arcsec. Blue spectra, covering the range $370-570 \mathrm{~nm}$, were obtained during three nights on May 10, July 11, and August 12, 2001 with the EEV10 CCD. Red spectra, covering the range 520-710 $\mathrm{nm}$, were obtained during two nights on August 27 and September 4, 2001 with the Tek5 CCD. Both red and blue portions were measured with a 632 lines $\mathrm{mm}^{-1}$ grating and $235 \mathrm{~mm}$ camera yielding $0.06 \mathrm{~nm} \mathrm{pixel}^{-1}$ for the blue spectra and $0.16 \mathrm{~nm}_{\text {pixel }}{ }^{-1}$ for the red spectra.

For each object, a series of exposures were taken with increasing exposure times from $60 \mathrm{~s}$ to $600 \mathrm{~s}$, to achieve both bright and faint emission lines without saturation. The list of objects observed may be found in Table 1 .

Each exposure was observed with the spectrograph slit aligned to the parallactic angle to avoid loss of light due to atmospheric differential refraction. We used a broad slit with a width of $7^{\prime \prime}$ to ensure as much light as possible from the object passed through the slit. Further discussion of slit loss may be found in Sect. 4. We used a narrower slit width of 1'.5 for radial velocity measurements, and observed $\mathrm{CuNe}$ arc lamps either before or after each observation.

IRAF software tasks in twodspec and image packages were used as follows for data reduction. The CCD's overscan region was used for bias level subtraction in each image. For each night, a set of either dome flats or sky flats were used to remove pixel-to-pixel variation, and to eliminate cosmetic defects associated with the instrument. Row-by-row wavelength calibration was performed on the two-dimensional image, to account for any variations in dispersion in the spatial direction.

The task apall was used to define an extraction aperture large enough to include all of the emission-lines spatially, trace the object along the dispersion direction, subtract the background, and extract the object. Background subtraction was conducted at each wavelength from the median of the background regions specified individually for each observation.

Flux calibration was carried out on all PNe with the general set of spectrophotometric standards (Stone \& Baldwin 1983; Baldwin \& Stone 1984; Massey et al. 1988; Oke 1990). Standards were observed at varying times and airmass throughout the night to derive a nightly extinction. The tasks standards, sensitivity, and calibrate, in order, were used for integrating the standard star fluxes over the calibrated bandpasses, defining a sensitivity function and extinction values, and flux calibrating each PNe.

Spectra taken with the narrow slit were used to determine systematic radial velocities for five of the target PNe (BoBn 1, DdDm 1, IC 5117, M 1-71, and NGC 6833). For M 1-5, additional spectroscopic observations on September 24, 2004 were obtained at the $4.2 \mathrm{~m}$ William Herschel Telescope with the ISIS red arm instrument. A spectral range of $607-713 \mathrm{~nm}$ was measured using the $500 \mathrm{~mm}^{-a m e r a}$ and 1200 lines $\mathrm{mm}^{-1}$, yielding a scale of $0.23 \mathrm{~nm} \mathrm{pixel}^{-1}$. A slit width of 0.5 was used. Radial velocites for all PNe were computed using the average wavelength shift of spectral lines relative to their rest wavelengths, with the IRAF task rvidlines.

\subsection{Images}

Compactness of target $\mathrm{PNe}$ is a basic requirement to ensure that the total flux of the nebula is included in our broad slit spectroscopic observations. Target $\mathrm{PNe}$ were chosen to have a diameter smaller than 4" in Acker et al. (1992; hereafter A92). Sizes of the targets were further checked using narrowband images taken at the INT and 2.6 Nordic Optical Telescope (NOT) of La Palma, and using HST archive images.

Images from NOT were obtained on September 9, 19, 20, and October 21, 1997, using the ALFOSC instrument Loral $2 \mathrm{k} \times 2 \mathrm{k} C \mathrm{CD}$, with a spatial scale of $0{ }^{\prime} 19$ per pixel. Narrowband filters were used to isolate the emission [NII] at $6583 \AA$ from the nearby hydrogen $\mathrm{H} \alpha$. For BoBn 1 , an [OIII] $5007 \AA$ image was also taken. Exposure times 


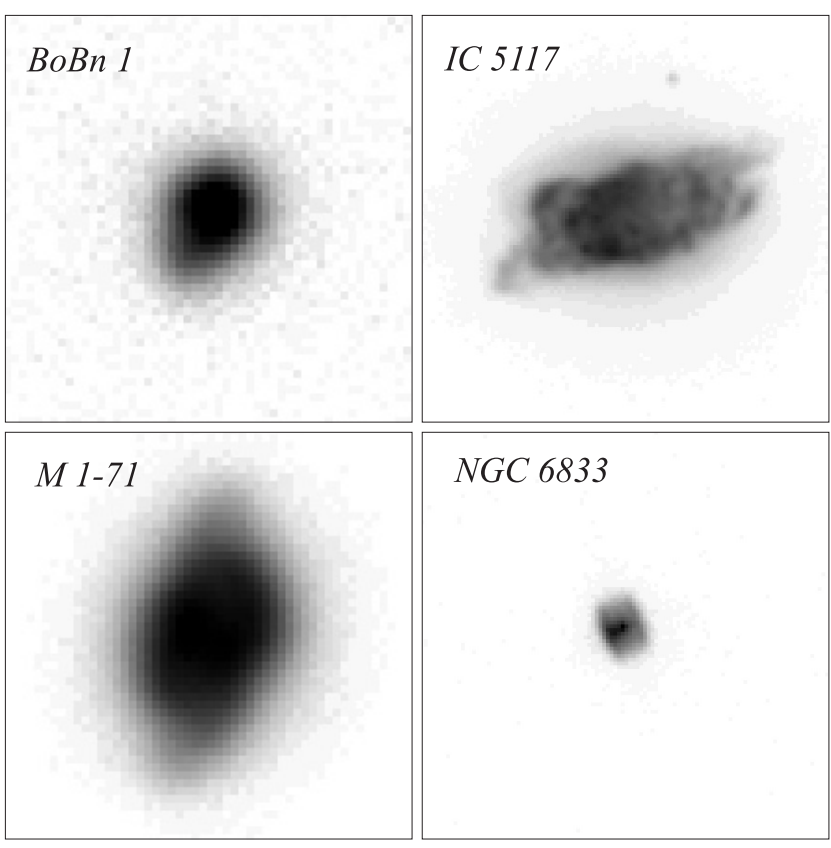

Fig. 1. [NII] images of four PNe discussed in this paper. North is at the top, East to the left. The field of view is $10^{\prime \prime} \times 10^{\prime \prime}$ for the NOT images of BoBn 1 and M 1-71, and $5^{\prime \prime} \times 5^{\prime \prime}$ for the HST images of IC 5117 and NGC 6833.

varied between $30 \mathrm{~s}$ and $10 \mathrm{~min}$, depending on the brightness of the nebula in each emission line and owing to the presence of thin clouds on some nights. The seeing varied between 0.7 and $1^{\prime \prime} .0$.

A $60 \mathrm{~s}$ image of M 1-5 was obtained with the prime-focus Wide Field Camera (WFC, pixel scale 0.'33) at the INT, on January 29, 2004. The filter used included both the $\mathrm{H} \alpha$ line and [NII] doublet. Seeing was 1". 4 .

Narrowband images of IC 5117 and NGC 6833 were retrieved from the HST public archive. They were taken with the PC CCD of the WFPC2 camera for programmes Nos. 6943 and 8307 on 1997 and 1999, respectively, using several narrowband filters including $\mathrm{H} \alpha$, [NII], and [OIII]. Exposure times range from $3 \mathrm{~s}$ to $10 \mathrm{~min}$.

\section{Morphology and sizes}

As is common for PNe, the largest extension of the nebulae shows up in the low excitation line of [NII], as compared to e.g. $\mathrm{H} \alpha$ and [OIII]. The [NII] images of four targets are displayed in Fig. 1. IC 5117 and NGC 6833 are well resolved by the HST. IC 5117 presents a highly elongated and clumpy morphology, possibly with a "multipolar" shape (cf. e.g. He 2-47 and M 1-37, Sahai 2000). NGC 6833 shows a bright nucleus from which two "boxy" lobes depart.

BoBn 1 and M 1-71 are also resolved in the ground-based images. BoBn 1 in the [NII] image shows a small protrusion toward the South-East. M 1-71 is larger, and appears to be composed of a bright "bulge" and a fainter disk-like structure extending approximately North-South.

DdDm 1 and M 1-5 are only marginally resolved in our ground-based images, and no details of their morphology can be appreciated. For this reason, they are not displayed in Fig. 1. DdDm 1 does not show any sign of elongation, while M 1-5 is slightly elliptical, as clearly shown in [OIII] and H $\beta$ HST images kindly made available by Matt Bobrowsky.

In Table 1 we list the sizes of the nebulae, measured as the diameter at the $10 \%$ level of the peak surface brightness, deconvolved by the instrumental resolution profile (seeing in the case of ground-based images). This method was introduced by Tylenda et al. (2003) and proved to be a good diameter estimator for these barely resolved images. For M 1-5, we used the HST image of Matt Bobrowsky.

\section{Analysis}

\subsection{Line fluxes and equivalent widths}

Reduced spectra were transferred to the STARLINK spectrum analysis program DIPSO (Howarth et al. 1996) to measure emission-line fluxes and equivalent widths. Local continua were fit with a first-degree polynomial on both sides of the emission-lines and subtracted from the spectra. Line fluxes were measured by integrating the continuum subtracted emission-lines between initial and ending points of emission features. Integration was performed to ensure we were deriving a "measured" flux as opposed to a fitted flux (e.g., from a Gaussian fit). Uncertainities were assigned to each flux line measurement based upon that frame's exposure time and flux calibration sensitivity.

Equivalent widths were measured with respect to local linear continua. Errors for each equivalent width measurement were derived by DIPSO based upon scatter of linear continuum fit and signal-to-noise of each emission-line.

Total absolute fluxes and equivalent widths listed in Tables 3 through 8 were computed from the weighted average of individual measurements in the various spectra. Their respective errors, also listed in the tables, were derived from the weighted standard deviation of the individual measurements for each emission line.

It should be noted that most of the errors listed in Tables 3-8 are "internal" errors which take into account the photon statistics of the observed PNe and standard stars, and the instrument and detector properties. In particular, this is the case when multiple observations for an object were all taken on a single night, and applies to the data of BoBn 1 (Table 3), DdDm 1 (Table 4), M 1-5 (Table 6), and M 1-71 (Table 7). When data are obtained in different nights, as for some emission lines of NGC 6833 and IC 5117 (noted in Tables 5 and 8), a better estimate of "external" systematic errors is obtained, for instance from the use of particular standard stars, or a nonperfect knowledge of the atmospheric extinction. Errors for multiple nights are larger and should be considered more realistic than for observations based on just a single night.

Another possible source of errors for the total fluxes of our target $\mathrm{PNe}$ are slit losses due to the finite width of the adopted slit. These have been kept as small as possible by selecting compact PNe and adopting a slit width of 7" for the spectroscopic observations. Slit losses have been estimated taking into account the size of each nebula in different emission lines from 
Table 2. Estimated total errors for all emission lines fluxes, and for available bright lines, namely $\mathrm{H} \beta, \mathrm{H} \alpha, 5007 \AA, 6583 \AA$, and $6678 \AA$.

\begin{tabular}{lcc}
\hline \hline $\begin{array}{l}\text { Object } \\
\text { name }\end{array}$ & $\begin{array}{c}\text { Total error } \\
\text { (all lines) }\end{array}$ & $\begin{array}{c}\text { Total error } \\
\text { (bright lines) }\end{array}$ \\
\hline Bobn 1 & $2.3 \%$ & $1.1 \%$ \\
DdDm 1 & $3.8 \%$ & $<1 \%$ \\
IC 5117 & $2.6 \%$ & $1.1 \%$ \\
M 1-5 & $2.3 \%$ & $<1 \%$ \\
M 1-71 & $3.1 \%$ & $2.8 \%$ \\
NGC 6833 & $5.2 \%$ & $<1 \%$ \\
\hline
\end{tabular}

the present images, seeing effects, slit width and its orientation with respect to the long axis of the resolved nebula, as well as centering and guiding errors. Slit losses are small in the case of the smaller nebulae, namely BoBn1, DdDm 1, and NGC 6833. Considering that seeing during the observations was generally not worse than 1.5 arcsec, slit losses for these nebulae are estimated to be below $0.5 \%$. The effect is slightly higher for M 1-5, were slit losses are estimated to be around $1.0 \%$. They are potentially larger for IC 5117, especially in [NII] where the nebula is most extended. However, except in M 1-71, our slit was oriented along the major axis of its elongated morphology, and thus no light from the outer regions of the nebula has escaped; slit losses are estimated to be at the same level as the first three nebulae, namely below $0.5 \%$. The only case in which slit losses are substantial (approximately $3 \%$ in [NII] and $2 \%$ in $\mathrm{H} \alpha$ ) is M 1-71, as the slit (oriented along the parallactic angle) was cutting the nebula through its short axis, a fact only partially compensated by the fact that the emission is highly centrally concentrated.

Including all the different sources of uncertainties discussed above, our estimates of the errors for the total fluxes to be associated with each individual PN are presented in Table 2. They are obtained by summing quadratically the errors due to slit losses with the average of the errors listed in Tables 3-8 for all lines in each individual PN, or for only the brightest ones $(\mathrm{H} \beta$, $\mathrm{H} \alpha, 5007 \AA, 6583 \AA$, and $6678 \AA$ ).

The conclusion is that narrowband images in bright emission lines can be calibrated photometrically using all the nebulae in our sample except for M 1-71 to a level of $1.4 \%$ or better, depending on the actual "standard" PN adopted. At the same level, secular variations of total flux of nebulae can be monitored by means of future spectrophotometric observations.

\subsection{Radial velocities}

Derived systemic velocities are presented in Table 1. Five out of six of our radial velocity measurements may be compared to previous literature, and in particular with the large compilation in Durand et al. (1998), which also includes the data quoted in A92. NGC 6833, M 1-5, and BoBn 1 are in good agreement with the velocity quoted in Durand et al. (1998). IC 5117 is a factor of $2 \sigma$ in disagreement, while the radial velocity measured more recently by Hyung et al. (2001) is consistent with our determination. Our M 1-71 radial velocity measurement is in good agreement with the older measurements quoted in A92, however, it does not match the new measurements of Durand et al. (1998) and their adopted value. Our high radial velocity for DdDm 1 is in excellent agreement with that reported by Barker \& Cudworth (1984).

\subsection{Physical conditions and chemical abundances}

The present spectra allow us to derive physical and chemical properties for four PNe: DdDm 1, IC 5117, NGC 6833, and M 1-71.

The same type of analysis as in Corradi et al. (1997) and Perinotto \& Corradi (1998) was adopted. The logarithmic extinction constants $c_{\beta}$ were computed from the Balmer decrement. Electron temperatures $T_{\mathrm{e}}([\mathrm{NII}])$ and $T_{\mathrm{e}}([\mathrm{OIII}])$ were computed from the standard auroral-to-nebular line ratios. As with electron density, in all nebulae where the [SII] 673.1/671.6 nm line ratio could be determined, it approaches its high density limit, implying $N_{\mathrm{e}}>10000 \mathrm{~cm}^{-3}$. For IC 5117 and DdDm 1, an estimate of $N_{\mathrm{e}}$ can be obtained from the [ClIII] $551.8 \mathrm{~nm}, 553.8 \mathrm{~nm}$ doublet. The $c_{\beta}$, and electron temperatures and densities are listed in Table 9.

Ionic abundances relative to hydrogen are computed given the temperature and densities, using the line fluxes relative to $\mathrm{H} \beta$ (Table 10). Total abundances and their errors (Table 11) were computed from line intensities as described in Corradi et al. (1997), using the ionization correction factor (ICF) from Kingsburgh \& Barlow (1994, hereafter, KB94). The low level of scatter in measurements for each emission-line flux did propagate into final uncertainties in the derived abundances of the order of 5 percent for helium and 25 percent for heavier elements.

\section{Discussion and conclusions}

The spectra obtained were used to infer some physical and chemical properties of the target nebulae. This analysis generally confirms and extends results obtained by other authors.

BoBn 1 and DdDm 1 are known to belong to the Galactic halo, owing to their high Galactic latitude, highly peculiar systemic radial velocity, and subsolar oxygen abundance (see also Barker \& Cudworth 1984; Howard et al. 1997; and Dinerstein et al. 2003). While our flux ratios for DdDm 1 do not agree well with Dinerstein et al. (2003), we observe no systematic effects between the three line ratios that correspond to our wavelength range.

An extensive spectroscopic study of IC 5117 was presented by Hyung et al. (2001), who show that this nebula must be very young owing to its high gas densities, ranging from $40000 \mathrm{~cm}^{-3}$ in the [ClIII] and [SII] emitting region up to $100000 \mathrm{~cm}^{-3}$ from line diagnostics involving $p^{3}$ electrons. The large density fluctuations found in this nebula are most likely related to the clumpy and highly asymmetrical morphology shown by HST images presented in this paper.

We found rather high gas densities in $M$ 1-71. Parthasarathy et al. (1998) classified the central star of M 1-71 in the class of "weak emission line stars" (WELS) or [WC]-PG1159 central stars. Our spectra confirm the presence 
Table 3. BoBn 1 fluxes and equivalent widths.

\begin{tabular}{lcccc}
\hline \hline $\begin{array}{l}\text { Wavelength } \\
(\AA)\end{array}$ & Ion & $\begin{array}{c}\log [\text { Flux }] \\
\left(\mathrm{erg} \mathrm{cm}^{-2} \mathrm{~s}^{-1}\right)\end{array}$ & $\begin{array}{c}\log [E W] \\
(\AA)\end{array}$ & $\begin{array}{c}\text { Number of } \\
\text { observations }\end{array}$ \\
\hline 4685.71 & $\mathrm{HeII}$ & $-13.103 \pm 0.024$ & $2.097 \pm 0.107$ & 3 \\
4861.29 & $\mathrm{H} \beta$ & $-12.425 \pm 0.011$ & $2.759 \pm 0.128$ & 4 \\
4959.52 & {$[\mathrm{OIII}]$} & $-12.358 \pm 0.005$ & $2.797 \pm 0.065$ & 4 \\
5007.57 & {$[\mathrm{OIII}]$} & $-11.877 \pm 0.006$ & $3.188 \pm 0.140$ & 4 \\
5754.59 & {$[\mathrm{NII}]$} & $-14.447 \pm 0.045$ & $1.000 \pm 0.105$ & 2 \\
5875.97 & $\mathrm{HeI}$ & $-13.278 \pm 0.019$ & $2.009 \pm 0.255$ & 4 \\
6300.30 & {$[\mathrm{OI}]$} & $-14.503 \pm 0.069$ & $0.986 \pm 0.050$ & 2 \\
6548.05 & {$[\mathrm{NII}]$} & $-13.296 \pm 0.014$ & $1.674 \pm 0.045$ & 4 \\
6562.85 & $\mathrm{H} \alpha$ & $-11.934 \pm 0.002$ & $2.959 \pm 0.059$ & 4 \\
6583.45 & {$[\mathrm{NII}]$} & $-12.828 \pm 0.012$ & $2.166 \pm 0.067$ & 4 \\
6678.15 & $\mathrm{HeI}$ & $-13.853 \pm 0.022$ & $1.585 \pm 0.206$ & 3 \\
7065.71 & $\mathrm{HeI}$ & $-13.723 \pm 0.038$ & $1.767 \pm 0.109$ & 3 \\
\hline
\end{tabular}

Table 4. DdDm 1 fluxes and equivalent widths.

\begin{tabular}{|c|c|c|c|c|}
\hline $\begin{array}{l}\text { Wavelength } \\
(\AA)\end{array}$ & Ion & $\begin{array}{c}\log [\text { Flux }] \\
\left(\mathrm{erg} \mathrm{cm}^{-2} \mathrm{~s}^{-1}\right)\end{array}$ & $\begin{array}{c}\log [E W] \\
(\AA)\end{array}$ & $\begin{array}{c}\text { Number of } \\
\text { observations }\end{array}$ \\
\hline 3726.19 & [OII] & $-11.759 \pm 0.001$ & $2.184 \pm 0.082$ & 2 \\
\hline 3750.15 & $\mathrm{H}_{12}$ & $-13.321 \pm 0.055$ & $0.719 \pm 0.045$ & 2 \\
\hline 3770.63 & $\mathrm{H}_{11}$ & $-13.257 \pm 0.043$ & $0.805 \pm 0.040$ & 3 \\
\hline 3797.90 & $\mathrm{H}_{10}$ & $-13.119 \pm 0.035$ & $0.938 \pm 0.052$ & 2 \\
\hline 3819.7 & $\mathrm{HeI}^{\star}$ & $-13.735 \pm 0.120$ & $0.267 \pm 0.102$ & 3 \\
\hline 3835.38 & $\mathrm{H}_{9}$ & $-12.969 \pm 0.031$ & $1.077 \pm 0.024$ & 4 \\
\hline 3868.71 & [NeIII] & $-12.313 \pm 0.009$ & $1.752 \pm 0.030$ & 3 \\
\hline 3889.05 & $\mathrm{H}_{8}$ & $-12.511 \pm 0.003$ & $1.562 \pm 0.068$ & 3 \\
\hline 3967.41 & [NeIII] & $-12.382 \pm 0.006$ & $1.724 \pm 0.015$ & 2 \\
\hline 4026.1 & $\mathrm{HeI}^{\star}$ & $-13.466 \pm 0.011$ & $0.672 \pm 0.033$ & 2 \\
\hline 4068.91 & CIII & $-13.530 \pm 0.024$ & $0.611 \pm 0.023$ & 4 \\
\hline 4101.74 & $\mathrm{H} \delta$ & $-12.400 \pm 0.017$ & $1.742 \pm 0.041$ & 4 \\
\hline 4340.47 & $\mathrm{H} \gamma$ & $-12.124 \pm 0.010$ & $2.096 \pm 0.034$ & 3 \\
\hline 4363.21 & [OIII] & $-13.096 \pm 0.012$ & $1.126 \pm 0.121$ & 2 \\
\hline 4387.93 & HeI & $-14.101 \pm 0.163$ & $0.135 \pm 0.253$ & 3 \\
\hline 4471.68 & $\mathrm{HeI}$ & $-13.098 \pm 0.021$ & $1.158 \pm 0.066$ & 3 \\
\hline 4861.20 & $\mathrm{H} \beta$ & $-11.794 \pm 0.001$ & $2.542 \pm 0.032$ & 3 \\
\hline 4959.52 & [OIII] & $-11.623 \pm 0.004$ & $2.699 \pm 0.036$ & 2 \\
\hline 5007.57 & [OIII] & $-11.145 \pm 0.0001$ & $3.034 \pm 0.120$ & 2 \\
\hline 5197.90 & {$[\mathrm{NI}]$} & $-14.144 \pm 0.002$ & $0.298 \pm 0.011$ & 2 \\
\hline 5269.20 & {$[\mathrm{KVI}]$} & $-13.714 \pm 0.019$ & $0.767 \pm 0.002$ & 2 \\
\hline 5517.72 & [ClIII] & $-14.459 \pm 0.132$ & $0.065 \pm 0.096$ & 2 \\
\hline 5537.89 & [ClIII] & $-14.339 \pm 0.158$ & $0.173 \pm 0.155$ & 2 \\
\hline 5754.59 & {$[\mathrm{NII}]$} & $-13.683 \pm 0.025$ & $0.906 \pm 0.040$ & 2 \\
\hline
\end{tabular}

${ }^{\star}$ HeI lines are a double blend.

of CIV $580.6 \mathrm{~nm}$ and 465.0 to $468.6 \mathrm{~nm}$ CIV-HeII broad stellar features in emission, but not of any OV and OVI lines.

To our knowledge, no abundance determinations are available for the nebula of M 1-71 prior to the present study. Our analysis confirms that, like in the other WELS and [WC] central stars of PNe, in spite of the fact that the central star of
M 1-71 is helium and carbon rich, no peculiar gas abundances are found in the nebula compared to the general sample of Galactic PNe (cf. Corradi \& Schwarz 1995).

NGC 6833 has a peculiar systemic radial velocity, deviating more than $50 \mathrm{~km} \mathrm{~s}^{-1}$ from the general Galactic rotation, (even assuming a large distance of $8-9 \mathrm{kpc}$ for this object). 
Table 5. IC 5117 fluxes and equivalent widths.

\begin{tabular}{|c|c|c|c|c|}
\hline $\begin{array}{l}\text { Wavelength } \\
(\AA)\end{array}$ & Ion & $\begin{array}{l}\log [\text { Flux }] \\
\left(\operatorname{erg~cm}^{-2} \mathrm{~s}^{-1}\right)\end{array}$ & $\begin{array}{l}\log [E W] \\
(\AA)\end{array}$ & $\begin{array}{c}\text { Number of } \\
\text { observations }\end{array}$ \\
\hline 3705.00 & $\mathrm{HeI}$ & $-13.661 \pm 0.155$ & $0.810 \pm 0.211$ & 2 \\
\hline 3712.75 & HeII & $-13.656 \pm 0.032$ & $0.806 \pm 0.122$ & 2 \\
\hline 3726.19 & {$[\mathrm{OII}]$} & $-12.337 \pm 0.004$ & $1.768 \pm 0.023$ & 2 \\
\hline 3734.37 & $\mathrm{H}_{13}$ & $-13.340 \pm 0.088$ & $0.493 \pm 0.261$ & 2 \\
\hline 3750.15 & $\mathrm{H}_{12}$ & $-13.254 \pm 0.021$ & $1.217 \pm 0.179$ & 2 \\
\hline 3770.63 & $\mathrm{H}_{11}$ & $-13.156 \pm 0.053$ & $1.502 \pm 0.051$ & 2 \\
\hline 3797.90 & $\mathrm{H}_{10}$ & $-13.014 \pm 0.005$ & $1.651 \pm 0.175$ & 2 \\
\hline 3819.70 & $\mathrm{HeI}^{\star}$ & $-13.700 \pm 0.001$ & $0.846 \pm 0.019$ & 2 \\
\hline 3835.38 & $\mathrm{H}_{9}$ & $-12.836 \pm 0.015$ & $1.767 \pm 0.016$ & 2 \\
\hline 3868.71 & [NeIII] & $-11.503 \pm 0.002$ & $2.871 \pm 0.071$ & 4 \\
\hline 3889.05 & $\mathrm{H}_{8}$ & $-12.482 \pm 0.013$ & $1.955 \pm 0.098$ & 3 \\
\hline 3967.41 & [NeIII] & $-11.844 \pm 0.003$ & $2.781 \pm 0.100$ & 4 \\
\hline 4068.91 & CIII & $-12.991 \pm 0.002$ & $1.382 \pm 0.063$ & 2 \\
\hline 4101.74 & $\mathrm{H} \delta$ & $-12.179 \pm 0.012$ & $2.387 \pm 0.134$ & 4 \\
\hline 4340.47 & $\mathrm{H} \gamma$ & $-11.876 \pm 0.003$ & $2.581 \pm 0.112$ & 4 \\
\hline 4363.21 & [OIII] & $-12.191 \pm 0.010$ & $2.262 \pm 0.077$ & 4 \\
\hline 4387.93 & $\mathrm{HeI}$ & $-13.878 \pm 0.152$ & $0.637 \pm 0.111$ & 2 \\
\hline 4471.68 & HeI & $-12.798 \pm 0.001$ & $1.783 \pm 0.024$ & 2 \\
\hline 4634.14 & NIII & $-13.576 \pm 0.036$ & $0.695 \pm 0.333$ & 4 \\
\hline 4640.64 & NIII & $-13.215 \pm 0.013$ & $0.947 \pm 0.291$ & 4 \\
\hline 4685.71 & HeII & $-12.359 \pm 0.001$ & $2.106 \pm 0.075$ & 4 \\
\hline 4713.38 & $\mathrm{HeI}^{\star}$ & $-13.036 \pm 0.003$ & $1.419 \pm 0.082$ & 4 \\
\hline 4724.30 & [NeIV] & $-14.154 \pm 0.057$ & $0.270 \pm 0.163$ & 4 \\
\hline 4740.18 & [ArIV] & $-12.661 \pm 0.001$ & $1.813 \pm 0.029$ & 4 \\
\hline 4861.20 & $\mathrm{H} \beta$ & $-11.380 \pm 0.020$ & $3.032 \pm 0.006$ & 6 \\
\hline 4921.93 & $\mathrm{HeI}$ & $-13.180 \pm 0.050$ & $1.019 \pm 0.063$ & 4 \\
\hline 4958.52 & [OIII] & $-10.629 \pm 0.023$ & $3.270 \pm 0.124$ & 5 \\
\hline 5007.57 & [OIII $]$ & $-10.131 \pm 0.017$ & $3.743 \pm 0.026$ & 2 \\
\hline 5191.80 & [ArIII] & $-14.152 \pm 0.159$ & $0.151 \pm 0.004$ & 4 \\
\hline 5197.90 & {$[\mathrm{NI}]$} & $-13.852 \pm 0.019$ & $0.488 \pm 0.116$ & 4 \\
\hline 5411.52 & HeII & $-13.276 \pm 0.042$ & $1.107 \pm 0.019$ & 4 \\
\hline 5517.72 & [ClIII] & $-14.084 \pm 0.025$ & $0.209 \pm 0.072$ & 4 \\
\hline 5537.89 & [ClIII $]$ & $-13.544 \pm 0.005$ & $0.801 \pm 0.007$ & 4 \\
\hline 5754.59 & [NII] & $-12.653 \pm 0.006$ & $1.650 \pm 0.005$ & 2 \\
\hline 5875.97 & HeI & $-11.909 \pm 0.024$ & $2.325 \pm 0.109$ & $9 \dagger$ \\
\hline 6300.30 & {$[\mathrm{OI}]$} & $-12.177 \pm 0.026$ & $1.881 \pm 0.050$ & $8 \dagger$ \\
\hline 6312.10 & [SIII] & $-12.606 \pm 0.039$ & $1.437 \pm 0.011$ & $9 \dagger$ \\
\hline 6363.77 & {$[\mathrm{OI}]$} & $-12.656 \pm 0.024$ & $1.453 \pm 0.038$ & $9 \dagger$ \\
\hline 6548.05 & {$[\mathrm{NII}]$} & $-11.733 \pm 0.032$ & $1.612 \pm 0.116$ & $9 \dagger$ \\
\hline 6562.85 & $\mathrm{H} \alpha$ & $-10.524 \pm 0.002$ & $2.887 \pm 0.223$ & $3 \dagger$ \\
\hline 6583.45 & [NII] & $-11.272 \pm 0.008$ & $2.312 \pm 0.184$ & $7 \dagger$ \\
\hline 6678.15 & $\mathrm{HeI}$ & $-12.366 \pm 0.003$ & $1.743 \pm 0.007$ & $9 \dagger$ \\
\hline 6716.47 & [SII] & $-12.958 \pm 0.006$ & $1.151 \pm 0.015$ & $9 \dagger$ \\
\hline 6730.85 & [SII] & $-12.612 \pm 0.008$ & $1.495 \pm 0.037$ & $9 \dagger$ \\
\hline 7065.71 & $\mathrm{HeI}$ & $-11.860 \pm 0.009$ & $2.200 \pm 0.044$ & $9 \dagger$ \\
\hline
\end{tabular}

${ }^{\star}$ HeI lines are a double blend.

$\dagger$ Measured values were determined from two nights of observation. 
Table 6. M 1-5 fluxes and equivalent widths.

\begin{tabular}{lcccc}
\hline \hline $\begin{array}{l}\text { Wavelength } \\
(\AA)\end{array}$ & Ion & $\begin{array}{c}\log [\text { Flux }] \\
\left(\mathrm{ergs} \mathrm{cm}^{-2} \mathrm{~s}^{-1}\right)\end{array}$ & $\begin{array}{c}\log [E W] \\
(\AA)\end{array}$ & $\begin{array}{c}\text { Number of } \\
\text { observations }\end{array}$ \\
\hline 5754.59 & {$[\mathrm{NII}]$} & $-13.355 \pm 0.098$ & $1.228 \pm 0.102$ & 4 \\
5875.97 & $\mathrm{HeI}$ & $-12.551 \pm 0.007$ & $2.029 \pm 0.062$ & 4 \\
6300.30 & {$[\mathrm{OI}]$} & $-13.273 \pm 0.032$ & $1.265 \pm 0.038$ & 4 \\
6312.10 & {$[\mathrm{SIII}]$} & $-13.685 \pm 0.034$ & $0.819 \pm 0.048$ & 3 \\
6363.77 & {$[\mathrm{OI}]$} & $-13.738 \pm 0.080$ & $0.779 \pm 0.060$ & 3 \\
6548.05 & {$[\mathrm{NII}]$} & $-12.086 \pm 0.002$ & $1.793 \pm 0.076$ & 4 \\
6562.85 & $\mathrm{H} \alpha$ & $-11.082 \pm 0.002$ & $2.817 \pm 0.029$ & 2 \\
6583.45 & {$[\mathrm{NII}]$} & $-11.595 \pm 0.001$ & $2.498 \pm 0.063$ & 4 \\
6678.15 & $\mathrm{HeI}$ & $-12.940 \pm 0.001$ & $1.572 \pm 0.032$ & 2 \\
6716.47 & {$[\mathrm{SII}]$} & $-13.497 \pm 0.005$ & $1.008 \pm 0.026$ & 2 \\
6730.85 & {$[\mathrm{SII}]$} & $-13.157 \pm 0.001$ & $1.332 \pm 0.016$ & 2 \\
7065.71 & $\mathrm{HeI}$ & $-12.513 \pm 0.002$ & $1.942 \pm 0.031$ & 4 \\
7135.80 & {$[\mathrm{ArIII}]$} & $-12.530 \pm 0.010$ & $1.919 \pm 0.045$ & 4 \\
\hline
\end{tabular}

Table 7. M 1-71 fluxes and equivalent widths. Note that an additional uncertainty of some $2-3 \%$ due to slit losses should be added to the errors quoted below (see text).

\begin{tabular}{lcccc}
\hline $\begin{array}{l}\text { Wavelength } \\
(\AA)\end{array}$ & Ion & $\begin{array}{c}\log [\text { Flux }] \\
\left(\mathrm{ergs} \mathrm{cm}^{-2} \mathrm{~s}^{-1}\right)\end{array}$ & $\begin{array}{c}\log [E W] \\
(\AA)\end{array}$ & $\begin{array}{c}\text { Number of } \\
\text { observations }\end{array}$ \\
\hline 3868.71 & {$[\mathrm{NeIII}]$} & $-12.683 \pm 0.032$ & $2.413 \pm 0.163$ & 2 \\
3967.41 & {$[\mathrm{NeIII}]$} & $-12.968 \pm 0.053$ & $2.295 \pm 0.161$ & 2 \\
4101.74 & $\mathrm{H} \delta$ & $-13.188 \pm 0.007$ & $2.027 \pm 0.158$ & 2 \\
4340.47 & $\mathrm{H} \gamma$ & $-12.803 \pm 0.036$ & $2.420 \pm 0.073$ & 2 \\
4363.21 & {$[\mathrm{OIII}]$} & $-13.554 \pm 0.019$ & $1.552 \pm 0.089$ & 2 \\
4471.68 & $\mathrm{HeI}$ & $-13.615 \pm 0.039$ & $1.744 \pm 0.041$ & 2 \\
4861.20 & $\mathrm{H} \beta$ & $-12.198 \pm 0.048$ & $2.911 \pm 0.228$ & 4 \\
4959.52 & {$[\mathrm{OIII}]$} & $-11.510 \pm 0.010$ & $3.207 \pm 0.045$ & 3 \\
5007.57 & {$[\mathrm{OIII}]$} & $-10.997 \pm 0.002$ & $3.645 \pm 0.147$ & 2 \\
5754.59 & {$[\mathrm{NII}]$} & $-13.210 \pm 0.011$ & $1.677 \pm 0.017$ & 2 \\
5875.97 & $\mathrm{HeI}$ & $-12.409 \pm 0.001$ & $2.394 \pm 0.061$ & 2 \\
6300.30 & {$[\mathrm{OI}]$} & $-12.625 \pm 0.007$ & $1.888 \pm 0.104$ & 2 \\
6312.10 & {$[\mathrm{SIII}]$} & $-13.597 \pm 0.001$ & $0.759 \pm 0.229$ & 2 \\
6363.77 & {$[\mathrm{OI}]$} & $-13.097 \pm 0.034$ & $1.598 \pm 0.050$ & 2 \\
6548.05 & {$[\mathrm{NII}]$} & $-11.961 \pm 0.011$ & $1.541 \pm 0.069$ & 2 \\
6562.85 & $\mathrm{H} \alpha$ & $-10.982 \pm 0.001$ & $2.590 \pm 0.052$ & $1 \ddagger$ \\
6583.45 & {$[\mathrm{NII}]$} & $-11.470 \pm 0.006$ & $2.469 \pm 0.035$ & 2 \\
6678.15 & $\mathrm{HeI}$ & $-12.751 \pm 0.006$ & $1.802 \pm 0.004$ & 2 \\
6716.47 & {$[\mathrm{SII}]$} & $-13.334 \pm 0.029$ & $1.067 \pm 0.093$ & 2 \\
6730.85 & {$[\mathrm{SII}]$} & $-13.027 \pm 0.012$ & $1.462 \pm 0.024$ & 2 \\
7065.71 & $\mathrm{HeI}$ & $-12.282 \pm 0.008$ & $2.193 \pm 0.035$ & 2 \\
\hline 5 & & & &
\end{tabular}

$\$$ Errors are based on one measurement.

The galactic coordinates and radial velocity measurements of NGC 6833 coincide well with the outer arm structure observed in HI within the range of $50^{\circ}<l<195^{\circ}$., $-5^{\circ}$. $<b<35^{\circ}$, and $-175 \mathrm{~km} \mathrm{~s}^{-1}<V_{\mathrm{LSR}}<-60 \mathrm{~km} \mathrm{~s}^{-1}$ (Wakker \& van Woerden 1991; Dwarakanath et al. 2002). Low oxygen, neon and nitrogen abundances (cf. also the data for the global sample of PNe summmarized in Corradi \& Schwarz 1995), suggests that NGC 6833 is a nebula belonging to a relatively old stellar population. The electron density lower limit of $10000 \mathrm{~cm}^{3} \mathrm{sug}-$ gests that this nebula is likely to be in an early evolutionary stage.

For NGC 6833, our chemical abundances are in good agreement with those listed in Perinotto (1991). Abundances and $T_{\mathrm{e}}$ ([OIII]) of IC 5117, NGC 6833, and DdDm 1 are also in good agreement with Mal'kov (1998), with abundances of He and heavier elements comparable within $<5 \%$, and effective temperatures deviating on average by $\sim 250 \mathrm{~K}$. 
Table 8. NGC 6833 fluxes and equivalent widths.

\begin{tabular}{|c|c|c|c|c|}
\hline $\begin{array}{l}\text { Wavelength } \\
(\AA)\end{array}$ & Ion & 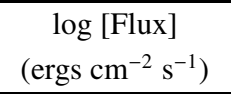 & $\begin{array}{c}\log [E W] \\
(\AA)\end{array}$ & $\begin{array}{c}\text { Number of } \\
\text { Observations }\end{array}$ \\
\hline 3697.15 & $\mathrm{H}_{17}$ & $-13.829 \pm 0.241$ & $-0.0003 \pm 0.109$ & $4 \dagger$ \\
\hline 3705.00 & $\mathrm{HeI}$ & $-13.288 \pm 0.081$ & $0.423 \pm 0.037$ & $4 \dagger$ \\
\hline 3712.75 & HeII & $-13.346 \pm 0.149$ & $0.401 \pm 0.043$ & $4 \dagger$ \\
\hline 3726.19 & [OII] & $-12.259 \pm 0.066$ & $1.358 \pm 0.012$ & $4 \dagger$ \\
\hline 3734.37 & $\mathrm{H}_{13}$ & $-12.977 \pm 0.116$ & $0.562 \pm 0.024$ & $4 \dagger$ \\
\hline 3750.15 & $\mathrm{H}_{12}$ & $-12.920 \pm 0.023$ & $0.959 \pm 0.004$ & $6 \dagger$ \\
\hline 3770.63 & $\mathrm{H}_{11}$ & $-12.812 \pm 0.024$ & $1.066 \pm 0.005$ & $6 \dagger$ \\
\hline 3797.90 & $\mathrm{H}_{10}$ & $-12.689 \pm 0.055$ & $1.184 \pm 0.003$ & $6 \dagger$ \\
\hline 3819.70 & $\mathrm{HeI}^{\star}$ & $-13.305 \pm 0.146$ & $0.573 \pm 0.076$ & $5 \dagger$ \\
\hline 3835.38 & $\mathrm{H}_{9}$ & $-12.540 \pm 0.030$ & $1.334 \pm 0.006$ & $6 \dagger$ \\
\hline 3868.71 & [NeIII] & $-11.439 \pm 0.020$ & $2.394 \pm 0.033$ & $8 \dagger$ \\
\hline 3889.05 & $\mathrm{H}_{8}$ & $-12.142 \pm 0.019$ & $1.692 \pm 0.024$ & $7 \dagger$ \\
\hline 3967.41 & {$[\mathrm{NeIII}]$} & $-11.761 \pm 0.001$ & $2.136 \pm 0.005$ & $8 \dagger$ \\
\hline 4026.10 & $\mathrm{HeI}^{\star}$ & $-13.025 \pm 0.013$ & $0.905 \pm 0.014$ & $5 \dagger$ \\
\hline 4068.91 & CIII & $-13.442 \pm 0.128$ & $0.481 \pm 0.117$ & $4 \dagger$ \\
\hline 4101.74 & $\mathrm{H} \delta$ & $-11.954 \pm 0.006$ & $1.988 \pm 0.001$ & $7 \dagger$ \\
\hline 4340.47 & $\mathrm{H} \gamma$ & $-11.673 \pm 0.037$ & $2.269 \pm 0.026$ & $8 \dagger$ \\
\hline 4363.21 & [OIII] & $-12.247 \pm 0.040$ & $1.715 \pm 0.056$ & $8 \dagger$ \\
\hline 4387.93 & HeI & $-13.601 \pm 0.042$ & $0.378 \pm 0.006$ & $5 \dagger$ \\
\hline 4471.68 & $\mathrm{HeI}$ & $-12.613 \pm 0.039$ & $1.369 \pm 0.030$ & $7 \dagger$ \\
\hline 4713.38 & $\mathrm{HeI}$ & $-13.244 \pm 0.009$ & $0.821 \pm 0.012$ & $6 \dagger$ \\
\hline 4740.18 & [ArIV] & $-13.447 \pm 0.034$ & $0.590 \pm 0.039$ & $5 \dagger$ \\
\hline 4861.20 & $\mathrm{H} \beta$ & $-11.315 \pm 0.012$ & $2.733 \pm 0.033$ & $7 \dagger$ \\
\hline 4921.93 & $\mathrm{HeI}$ & $-13.246 \pm 0.034$ & $0.785 \pm 0.050$ & $4 \dagger$ \\
\hline 4959.52 & [OIII] & $-10.886 \pm 0.007$ & $3.003 \pm 0.023$ & $4 \dagger$ \\
\hline 5007.57 & [OIII $]$ & $-10.401 \pm 0.001$ & $3.439 \pm 0.074$ & $2 \dagger$ \\
\hline 5191.80 & [ArIII] & $-14.275 \pm 0.177$ & $-0.150 \pm 0.186$ & 2 \\
\hline 5537.89 & [ClIII $]$ & $-13.935 \pm 0.082$ & $0.243 \pm 0.098$ & 2 \\
\hline 5754.59 & {$[\mathrm{NII}]$} & $-13.049 \pm 0.047$ & $1.121 \pm 0.017$ & 6 \\
\hline 5875.97 & $\mathrm{HeI}$ & $-12.038 \pm 0.002$ & $2.112 \pm 0.011$ & 3 \\
\hline 6300.30 & {$[\mathrm{OI}]$} & $-12.841 \pm 0.268$ & $1.342 \pm 0.272$ & 3 \\
\hline 6363.77 & {$[\mathrm{OI}]$} & $-13.245 \pm 0.006$ & $0.966 \pm 0.080$ & 3 \\
\hline 6548.05 & {$[\mathrm{NII}]$} & $-12.262 \pm 0.007$ & $1.247 \pm 0.038$ & 4 \\
\hline 6562.85 & $\mathrm{H} \alpha$ & $-10.752 \pm 0.001$ & $2.838 \pm 0.051$ & $1+$ \\
\hline 6583.45 & {$[\mathrm{NII}]$} & $-11.865 \pm 0.003$ & $1.867 \pm 0.021$ & 4 \\
\hline 6678.15 & $\mathrm{HeI}$ & $-12.620 \pm 0.007$ & $1.598 \pm 0.028$ & 4 \\
\hline 6716.47 & [SII] & $-13.888 \pm 0.033$ & $0.329 \pm 0.035$ & 3 \\
\hline 6730.85 & [SII] & $-13.531 \pm 0.029$ & $0.700 \pm 0.001$ & 3 \\
\hline 7065.71 & $\mathrm{HeI}$ & $-12.130 \pm 0.003$ & $2.123 \pm 0.009$ & 3 \\
\hline
\end{tabular}

* HeI lines are a double blend.

$\dagger$ Measured values were determined from two nights of observation.

$\ddagger$ Errors are based on one measurement.

By comparing our abundance measurements with KB94 average abundance values for a particular class of $\mathrm{PNe}$, we see that IC 5117 and M 1-71 fall within the range for non-type 1 PNe. Our abundance measurements for NGC 6833 (except $\mathrm{He}$ ) are all significantly below the average KB94 value for non-type 1 Pne; $\mathrm{O}, \mathrm{N}$, and $\mathrm{Ne}$ abundances are a factor of 3.8, 2.5, and 3.6 below, respectively and $\mathrm{Ar}$ and $\mathrm{S}$ are even further below. If NGC 6833 is associated with the outer arm, then its abundance measurements would be an indicator of the outer arm's metallicity, which has been estimated to be lower than the inner disk (Digel er al. 1990). However, we cannot exclude the possibility that it belongs to the Galactic halo.

For four PNe, IC 5117, M 1-5, M 1-71, and NGC 6833, $\mathrm{H} \beta$ flux falls within one sigma of $\mathrm{A} 92$ reported $\mathrm{H} \beta$ fluxes. 
Table 9. Logarithmic extinction constant $c_{\beta}$, and electron temperatures and densities. Errors on temperatures are within 500 Kelvin.

\begin{tabular}{lccccc}
\hline \hline $\begin{array}{l}\text { Object } \\
\text { name }\end{array}$ & $c_{\beta}$ & $\begin{array}{c}T_{\mathrm{e}}([\mathrm{OIII}]) \\
(\mathrm{K})\end{array}$ & $\begin{array}{c}T_{\mathrm{e}}([\mathrm{NII}]) \\
(\mathrm{K})\end{array}$ & $\begin{array}{c}N_{\mathrm{e}}([\mathrm{SII}]) \\
\left(\mathrm{cm}^{-3}\right)\end{array}$ & $\begin{array}{c}N_{\mathrm{e}}([\mathrm{ClIII}]) \\
\left(\mathrm{cm}^{-3}\right)\end{array}$ \\
\hline DdDm1 & - & 12200 & - & - & 3700 \\
IC 5117 & 1.30 & 12500 & 13500 & - & 38000 \\
NGC 6833 & 0.34 & 13200 & 15400 & $>10000$ & - \\
M 1-71 & 2.44 & 9800 & 10200 & $>10000$ & - \\
\hline
\end{tabular}

Table 10. Ionic abundances relative to hydrogen.

\begin{tabular}{|c|c|c|c|c|c|c|c|c|c|c|c|}
\hline Object & $\mathrm{HeI}$ & HeII & OI & OII & OIII & NI & NII & SII & SIII & NeIII & ArIV \\
\hline DdDm 1 & $1.04 \mathrm{e}-1$ & - & - & - & $8.6 e-5$ & - & - & - & - & $1.5 \mathrm{e}-5$ & - \\
\hline IC 5117 & $9.33 \mathrm{e}-2$ & $1.0 \mathrm{e}-2$ & $5.4 \mathrm{e}-6$ & $1.3 e-5$ & $2.9 \mathrm{e}-4$ & $1.3 \mathrm{e}-6$ & $5.9 \mathrm{e}-6$ & $1.8 \mathrm{e}-7$ & $2.0 \mathrm{e}-6$ & - & - \\
\hline M 1-71 & $1.17 \mathrm{e}-1$ & - & $1.6 \mathrm{e}-5$ & - & $5.0 \mathrm{e}-4$ & - & $2.1 \mathrm{e}-5$ & $3.4 \mathrm{e}-7$ & $1.8 \mathrm{e}-6$ & $1.5 \mathrm{e}-4$ & - \\
\hline NGC 6833 & $9.05 \mathrm{e}-2$ & - & $1.3 e-6$ & $4.5 \mathrm{e}-6$ & $1.2 \mathrm{e}-4$ & - & $2.0 \mathrm{e}-6$ & $3.0 \mathrm{e}-8$ & - & $3.4 \mathrm{e}-5$ & $4.7 \mathrm{e}-8$ \\
\hline
\end{tabular}

Table 11. Total abundances and ionization correction factors (ICF).

\begin{tabular}{lccccccccccc}
\hline \hline Object & $\mathrm{He}$ & $\mathrm{O}$ & $\mathrm{ICF}[\mathrm{O}]$ & $\mathrm{N}$ & $\mathrm{ICF}[\mathrm{N}]$ & $\mathrm{S}$ & $\mathrm{ICF}[\mathrm{S}]$ & $\mathrm{Ne}$ & $\mathrm{ICF}[\mathrm{Ne}]$ & $\mathrm{Ar}$ & $\mathrm{ICF}[\mathrm{Ar}]$ \\
\hline IC 5117 & $1.04 \mathrm{e}-1$ & $3.2 \mathrm{e}-4$ & 1.07 & $1.5 \mathrm{e}-4$ & 25.51 & $4.4 \mathrm{e}-6$ & 2.07 & - & - & - & - \\
M 1-71 & $1.17 \mathrm{e}-1$ & $5.0 \mathrm{e}-4$ & 1.00 & - & - & - & - & $1.5 \mathrm{e}-4$ & 1.00 & - & - \\
NGC 6833 & $9.05 \mathrm{e}-2$ & $1.3 \mathrm{e}-4$ & 1.00 & $5.6 \mathrm{e}-5$ & 27.98 & $6.3 \mathrm{e}-8$ & 2.13 & $3.5 \mathrm{e}-5$ & 1.04 & $4.9 \mathrm{e}-8$ & 1.04 \\
\hline
\end{tabular}

BoBn 1 and DdDm 1 are greater than three sigma from A92 $\mathrm{H} \beta$ measurements. This discrepancy between our measurements and A92 may be due to underestimated uncertainities within the A92 catalogue, which has been recently noted in Ruffle et al. (2004). However, if the flux of BoBn 1 and DdDm 1 accurately represent intrinsic variability, then this calls for future monitoring of these sources.

In conclusion, following the work done in the southern hemisphere by Dopita \& Hua (1997), the present data provide an extension to the northern hemisphere of new emission-line standards for narrowband imaging. Further work of this kind is needed in order to have more complete coverage in right ascension and radial velocities.

Acknowledgements. The authors thank Begoña García for one night of service observations, Denise Gonçalves for further observations, Danny Lennon for his thoughtful help in data reduction and analysis, Remington P.S. Stone for valuable comments, and the referee for a number of helpful insights.

\section{References}

Acker, A., Ochsenbein, F., Stenholm, B., et al. 1992, Strasbourg-ESO Catalogue of Galactic Planetary Nebulae, ESO, Garching, SECAT Baldwin, J. A., \& Stone, R. P. S. 1984, MNRAS, 206, 241

Barker, T. 1978, ApJ, 264, 594

Barker, T., \& Cudworth, K. M. 1984, ApJ, 278, 610

Cahn, J. H., Kaler, J. B., \& Stanghellini, L. 1992, A\&AS, 94, 399

Capriotti, E. R., \& Daub, C. T. 1960, ApJ, 132, 677

Corradi, R. L. M., \& Schwarz, H. E. 1995, A\&A, 293, 871

Corradi, R. L. M., Perinotto, M., Schwarz, H. E., \& Claeskens, J.-F. 1997, A\&A, 322, 975
Digel, S., Thaddeus, P., \& Bally, J. 1990, ApJ, 357, L29

Dinerstein, H. L., Richter, M. J., Lacy, J. H., \& Sellgren, K. 2003, AJ, 125,265

Dopita, M. A., \& Hua, C. T. 1997, ApJS, 108, 515

Durand, S., Acker, A., \& Zijlstra, A. 1998, A\&A, 132, 13

Dwarakanath, K. S., Carilli, C. L., \& Goss, W. M. 2002, ApJ, 567, 940

Howard, J. W., Henry, R. B. C., \& McCartney, S. 1997, MNRAS, 284, 465

Howarth, I. D., Murray, J., Mills, D., \& Berry, D. S. 1996, STARLINK User Note SUN 50, Rutherford Appleton Laboratory/CCLRC

Hyung, S., Aller, L. H., Feibelman, W. A., \& Lee, S.-J. 2001, ApJ, 563,889

Johnson, H. L., \& Harris, D. L. 1954, ApJ, 120, 196

Kingsburgh, R. L., \& Barlow, M. J. 1994, MNRAS, 271, 257

Landolt, A. U. 1992, AJ, 104, 340

Liller, W. 1955, ApJ, 122, 240

Liller, W., \& Aller, L. H. 1954, ApJ, 120, 48

Mal'kov, Yu. F. 1998, ARep, 42, 293

Massey, P., Strobel, K., Barner, J. V., \& Anderson, E. 1988, ApJ, 328, 315

Miller, J. S., \& Mathews, W. G. 1972, ApJ, 172, 593

O'Dell, C. R. 1963, ApJ, 138, 1018

Oke, J. B. 1990, AJ, 99, 1621

Parthasarathy, M., Acker, A., \& Stenholm, B. 1998, A\&A, 329, L9

Perinotto, M. 1991, ApJS, 76, 687

Perinotto, M., \& Corradi, R. L. M. 1998, A\&A, 332, 721

Ruffle, P. M. E., Zijlstra, A. A., Walsh, J. R., et al. 2004, MNRAS, 353,796

Sahai, R. 2000, ApJ, 537, L43

Stone, R. P. S., \& Baldwin, J. A. 1983, MNRAS, 204, 347

Tylenda, R., Siódmak, N., Górny, S. K., Corradi, R. L. M., \& Schwarz, H. E. 2003, A\&A, 405, 627

Wakker, B. P., \& van Woerden, H. 1991, AAP, 250, 509 